\title{
Altered Expression Pattern of CD55 and CD59 on Red Blood Cells in Anemia of Chronic Kidney Disease
}

\author{
Lama Al-Faris $^{a}$ Salah Al-Humood ${ }^{a}$ Fatma Behbehani $^{b} \quad$ Husam Sallam $^{c}$ \\ a Department of Pathology, Faculty of Medicine, Kuwait University, Safat, and Departments of \\ ${ }^{\mathrm{b}}$ Laboratory Medicine, and ${ }^{\mathrm{C}}$ Medicine, Nephrology Unit, Amiri Hospital, Kuwait City, Kuwait
}

\section{Significance of the Study}

- In this study, patients with chronic kidney disease (CKD) had an altered expression of CD55 and CD59 on red blood cells, which might be a contributory factor to the pathophysiology of anemia in CKD. This finding could lead to new insights in the development of new treatment modalities for these patients.

\section{Keywords}

CD55 - CD59 - Anemia · Chronic kidney disease

\begin{abstract}
Objective: The aim of this study was to investigate the expression pattern of CD55 and CD59 on red blood cells (RBCs) in anemic chronic kidney disease (CKD) patients, and factors that might influence their expression. Subjects and Methods: Nighty-one adult anemic CKD patients and 80 healthy controls (HCs) were enrolled. Anemic CKD patients were divided into 3 subgroups based on receiving erythropoietin and renal replacement therapies. Flow cytometric analysis of CD55 and CD59 expression was performed on RBCs from blood samples obtained from CKD patients and HCs. Results: CD59 deficiency was significantly higher among CKD patients than HCs ( $n=68,74.7 \%$, vs. $n=13,16.3 \%$, respectively; $p<0.001$ ). The median proportions of CD55- and
\end{abstract}

\section{KARGER}

E-Mail karger@karger.com www.karger.com/mpp

\section{() 2017 The Author(s) \\ Published by S. Karger AG, Basel \\ Karger \\ Open access}

This is an Open Access article licensed under the Creative Commons Attribution-NonCommercial-4.0 International License (CC BY-NC) (http://www.karger.com/Services/OpenAccessLicense), applicable to the online version of the article only. Usage and distribution for commercial purposes requires written permission.
CD59-deficient RBCs in CKD patients were significantly higher compared to HCs (0.34 vs. 0.15 , and 4.3 vs. $2.0, p<0.001$ and $p<0.001$, respectively). The mean fluorescence intensity (MFI) of CD55 and CD59 expression was significantly lower in CKD patients compared to HCs (1.2 vs. 2.8, and 17.0 vs. $20.3, p<0.04$ and $p<0.001$, respectively). The hemoglobin level was inversely correlated with the proportions of CD55- and CD59-deficient RBCs $(r=-0.37, p<0.001$, and $r=$ $-0.22, p<0.02$, respectively). The number of CD59-deficient patients was significantly different between the 3 subgroups of CKD patients $(p=0.001)$, and a significant difference was present in the MFI of CD55 and CD59 expression among the 3 subgroups ( $p=0.04$ and $p=0.03$, respectively). Conclusion: The expression pattern of CD55 and CD59 on RBCs is altered in anemic CKD patients, which could play a role in the pathogenesis of anemia in CKD.

(c) 2017 The Author(s) Published by S. Karger AG, Basel 


\section{Introduction}

Anemia is one of the most characteristic manifestations of chronic kidney disease (CKD) [1]. It is the most common of the hypoproliferative anemias resulting from organ dysfunction. Previous epidemiological studies had shown that $48-68 \%$ of predialysis end-stage renal disease patients had a hematocrit $<0.30$, and $51 \%$ of these had a hematocrit $<25 \%[1,2]$. Anemia is not as common in earlier stages of CKD; patients with stage III disease have a prevalence of concurrent anemia of 5.2\%, whereas those with stage IV disease have a prevalence of concurrent anemia of $44.1 \%$ [2]. The degree of anemia appears to be proportional to the degree of impairment of renal function, because the ability of the kidneys to secrete erythropoietin (EPO) deteriorates as renal function worsens; however, a direct correlation to the degree of renal dysfunction varies greatly between patients [3].

In addition to the underproduction of EPO secondary to a decrease in the number of renal cortical cells, experimental and clinical observations revealed some of the other pathophysiologic mechanisms responsible for the etiology of anemia, including uremic-induced inhibitors of erythropoiesis, disordered iron homeostasis, shortened red blood cell (RBC) survival time, and concomitant blood loss secondary to uremic platelet dysfunction [3]. In patients undergoing dialysis, dialysis itself might contribute to the etiology of anemia through blood loss in the dialysis circuit due to hemolysis that could occur if there are problems with the dialysate (e.g., temperature problems, contamination with aluminum, fluoride, copper, chlorine, or chloramine) and deficiency of the dialyzable folate [4]. Secondary hyperparathyroidism also contributes to anemia in patients with CKD through suppression of the bone marrow $[4,5]$.

Survival of RBCs is also mildly to moderately decreased in patients with renal insufficiency [6]. In the setting of anemia, RBCs become less deformable and are more susceptible to mechanical destruction and clearance by macrophages [7]. Metabolically, uremia causes RBCs to have a decreased activity of enzymes involved in the hexosemonophosphate shunt and decreases ATPase activity [7]. This leads to increased susceptibility to oxidative stress, abnormal membrane permeability, and increased RBC osmotic fragility that can lead to a decreased RBC life span [8]. Hemodialysis also introduces RBC toxins such as copper, formaldehyde, chlorine, nitrates, and chloramine, which can damage RBCs and decrease survival [8]. The mechanical process of dialysis can also lead to RBC fragmentation [8].

CD55 and CD59 Expression in Anemia of CKD
Normal RBCs are well equipped to withstand damage by activated complement [9]. The complement system includes soluble and cell membrane-bound proteins, which circulate in the plasma in inactive forms, and can be activated through the classical pathway, alternative pathway, and lectin pathway [9]. These 3 pathways lead to C3/C5 convertase enzyme and the terminal pathway pore-like membrane attack complex formation, which leads to cell lysis [10]. RBCs are normally protected from complement-mediated lysis by the action of surface complement regulatory proteins, including decay-accelerating factor (CD55), and membrane inhibitor of reactive lysis (CD59), which belong to the group of proteins that are linked to the cell membrane via a GPI (glycosylphosphatidylinositol) anchor [9]. CD55 inhibits the aggregation of C3 and C5 convertases of the classical and alternative pathways, and thereby regulates the complement cascade at the $\mathrm{C} 3$ step, while CD59 limits the polymerization of C9 in the membrane C5b-9 complex [11].

Although the role of CD55 and CD59 deficiencies is well understood in paroxysmal nocturnal hemoglobinuria $(\mathrm{PNH})$, an acquired hemolytic anemia characterized by augmented sensitivity to complement-mediated lysis, limited information is available on the role of complement in RBC lysis in CKD, and on the influence of the EPO level and other proinflammatory cytokines on their expression on RBCs from patients with CKD [12]. Hence, the objective of this study was to explore the patterns of CD55 and CD59 expression on RBCs in patients with $\mathrm{CKD}$, and their relationship with EPO levels and the inflammatory status of the disease, as expressed by the level of C-reactive protein (CRP).

\section{Materials and Methods}

\section{Study Population}

Ninety-one patients ( 49 males, 42 females) and 80 age- and sexmatched healthy controls (HCs) were enrolled in this study cohort. The expression pattern of CD55 and CD59 on RBCs of all enrolled CKD patients, as 1 cohort, was compared to that on RBCs of HCs. The effect of EPO therapy and renal replacement therapy (RRT) on the expression pattern of CD55 and CD59 on RBCs was also studied and compared among the anemic CKD patients, who were divided into 3 subgroups: group $\mathrm{A}(n=30)$, treated with EPO therapy but not on RRT; group B $(n=30)$, treated with EPO therapy and RRT, and group C $(n=31)$, who were neither on EPO therapy nor on RRT. In this study, the WHO definition of anemia was used, i.e., males and females whose hemoglobin levels are $<130 \mathrm{~g} / \mathrm{L}$ and $<120 \mathrm{~g} / \mathrm{L}$, respectively. The patients were recruited from Al-Amiri Hospital, and blood samples were collected over a 12-month period (April 2015 to March 2016). None of the patients had a history of blood transfusion in the last 3 months prior to sample col- 
lection. The study was approved by the Research Ethics Committee of the Ministry of Health, and informed written consent was obtained from all participating CKD patients and HCs. Clinical data of all patients were obtained from their medical records.

\section{Flow Cytometric Analysis of CD55 and CD59 on RBCs}

Peripheral venous blood samples $(4.5 \mathrm{~mL})$ were collected from all enrolled subjects in test tubes containing EDTA (ethylenediaminetetraacetic acid) as an anticoagulant. An indirect immunofluorescence method was performed as described in the package insert of the Redquant CD55/CD59 kit (BioCytex, Marseille, France). The kit contained CD55 (clone 29D9) and CD59 (clone 2.24), monoclonal antibodies, fluorescein isothiocyanate (FITC)conjugated goat anti-mouse Ig reagent, precalibrated $\alpha$-beads for CD55 analysis and $\beta$-beads for CD59 analysis on RBCs, and phosphate-buffered saline (PBS). Briefly, $20 \mu \mathrm{L}$ of diluted EDTA whole blood $(1: 150)$ was incubated with $20 \mu \mathrm{L}$ of CD55 or CD59 for 10 $\mathrm{min}$ at room temperature. Two additional test tubes were used for each series, containing $40 \mu \mathrm{L}$ of precalibrated $a$-beads or precalibrated $\beta$-beads, respectively. Test tubes containing stained whole blood and beads were incubated with $20 \mu \mathrm{L}$ of FITC reagent for an additional $10 \mathrm{~min}$. After the staining procedure, samples were diluted with PBS $(2 \mathrm{~mL})$ and stored at $4^{\circ} \mathrm{C}$ for up to $4 \mathrm{~h}$ until measurement. Flow cytometric analysis was performed within $8 \mathrm{~h}$ of sample collection according to the Redquant kit manufacturer's instructions (BioCytex).

The RBCs were analyzed for CD55 and CD59 expression using the Redquant kit according to manufacturer's instructions. The Cytomics FC 500 Beckman Coulter Flow Cytometer instrument (Beckman Coulter Inc., Brea, CA, USA) is available in the Research Core Facility laboratories in the Faculty of Medicine, Kuwait University. RBCs were gated on the basis of their forward and side scatter characteristics with the use of logarithmic amplification and 10,000 events were analyzed for each antibody. To set the interpretation cursors for CD55 and CD59, the mean fluorescence intensity (MFI) of pathological regions, the $\alpha$ - and $\beta$-beads, was displayed as the geometrical mean (GeoMean) $r$. The beads were used to set a standardized cursor in the fluorescence histogram (CD55 cursor and CD59 cursor markers), the left limit of which was set in the first channel and right limit corresponded to a standardized fluorescence channel position. The CD55 and CD59 cursor markers were determined by the following equations as stated in the manufacturer's data sheet: [ $\alpha \mathrm{FI}=$ " $\alpha$ " $\times \alpha$-bead MFI] for the CD55 cursor, and $[\beta F I=$ " $\beta$ " $\times \beta$-bead MFI] for the CD59 cursor, where " $\alpha$ " and " $\beta$ " values are indicated in the assay value insert included in the kit. Both standardized cursors allowed the percentage of CD55- and CD59-deficient cells to be determined. Thus, 2 parameters were obtained: the percentage of deficient CD55 and CD59 RBCs. The presence of a deficient clone was suspected when the pathological region contained more than 3\% antigen-deficient cells. The strength of CD59 and CD55 expression, as evident by their MFI, on RBCs in CKD patients and HCs was also evaluated.

\section{Measurement of EPO Levels}

Peripheral venous blood samples were collected from all enrolled CKD patients and HCs in plain tubes. Blood samples were centrifuged at 5,000 $\mathrm{g}$ for $15 \mathrm{~min}$, and serum samples were transferred into coded tubes and frozen at $-80^{\circ} \mathrm{C}$ until the time of the assay. Enzyme-linked immunosorbent assay was used to measure EPO levels (eBioscience, San Diego, CA, USA).
Table 1. Demographic data and laboratory findings of CKD patients and $\mathrm{HCs}$

\begin{tabular}{lcc}
\hline Parameters & $\begin{array}{l}\text { CKD patients } \\
(n=91)\end{array}$ & $\begin{array}{l}\text { HCs } \\
(n=80)\end{array}$ \\
\hline Age, years & $56.6 \pm 13$ & $51.4 \pm 11$ \\
Gender & & \\
$\quad$ Male & $49(54)$ & $41(51)$ \\
Female & $42(46)$ & $39(49)$ \\
Hb, g/L & $105.2 \pm 12.3$ & $141 \pm 14$ \\
MCV, fL & $87.1 \pm 4.5$ & $86 \pm 4.2$ \\
RDW, \% & $17.3 \pm 2.5$ & $13 \pm 1.3$ \\
Reticulocyte count, $\times 10^{9} / \mathrm{L}$ & $56(31-78)$ & $70(52-98)$ \\
LDH, IU/L & $191(153-255)$ & $140(110-200)$ \\
S-bilirubin, $\mu \mathrm{mol} / \mathrm{L}$ & $13(11-22)$ & $15(12-23)$ \\
CRP, mg/L & $7.6(4.6-32.4)$ & $3.3(0.9-6.1)$ \\
Creatinine, $\mu \mathrm{mol} / \mathrm{L}$ & $546(380-869)$ & $62(55-92)$ \\
BUN, mmol/L & $18.8(16-25.8)$ & $4.2(2.2-8.3)$ \\
S-EPO, MIU/mL & $28(15-32)$ & nd \\
\hline
\end{tabular}

Values are the mean $\pm \mathrm{SD}, n(\%)$, or median (IQR).

$\mathrm{CKD}$, chronic kidney disease; WBC, white blood cell count; $\mathrm{Hb}$, hemoglobin; MCV, mean cell volume; RDW, red cell distribution width; BUN, blood urea nitrogen; $\mathrm{LDH}$, lactate dehydrogenase; CRP, C-reactive protein; EPO, erythropoietin; nd, not done.

Measurement of Parameters of Hemolysis and Inflammation

Biochemical and hematologic parameters were measured as part of the patients' routine care, including complete blood cell count, reticulocyte count (Beckman Coulter Hematology Analyzer), lactate dehydrogenase (LDH), and CRP (Beckman Coulter IMMAGE Immunochemistry System).

\section{Statistical Analyses}

Data were analyzed using IBM SPSS Statistics, version 23 (IBM, Armonk, NY, USA). Quantitative variables were analyzed using descriptive statistics that included measures of location (means plus standard deviations) and medians (interquartile ranges, IQR; 25 th and 75th percentiles) for parametric and nonparametric variables, respectively. The bivariate distribution was evaluated for normality using the abovementioned standard descriptive statistical methods and by examining distribution plots. Parametric and nonparametric measures of comparison were used for symmetrical and skewed variables, respectively. Comparisons among the patient groups (CKD patients and HCs) were tested using the independent-sample $t$ test. Correlations were tested using standard statistical methods depending on data distribution, the type of outcome measures, and the type of predictors, including the following: $\chi^{2}$ test to determine whether there is a significant difference between CD55 and CD59 deficiency states among CKD patients and HC groups, analysis of variance (ANOVA) to compare continuous outcome measures among the $3 \mathrm{CKD}$ patient groups, Mann-Whitney test to measure correlations between categorical predictors and nonparametric continuous outcome variables, and linear regression to test the associations between CD55- and CD59-related variables and parameters of hemolysis in CKD patients. A $p$ value $<0.05$ (2-tailed) was considered statistically significant. 
Table 2. Comparison between CD55 and CD59 expression patterns among CKD patients and HCs

\begin{tabular}{lccc}
\hline Parameters & $\begin{array}{l}\text { CKD patients } \\
(n=91)\end{array}$ & $\begin{array}{l}\text { HCs } \\
(n=80)\end{array}$ & $p$ \\
\hline CD55-deficient subjects & $0(0)$ & $0(0)$ & 1.000 \\
CD59-deficient subjects & $68(74.7)$ & $13(16.3)$ & $<0.001^{*}$ \\
Proportion of CD55-deficient RBCs, \% & $0.34(0.16-0.46)$ & $0.15(0.04-0.19)$ & $<0.001^{*}$ \\
Proportion of CD59-deficient RBCs, \% & $4.3(2.8-4.4)$ & $2(0.7-2.8)$ & $<0.001^{*}$ \\
MFI of CD55-positive cells & $1.2 \pm 0.13$ & $2.8 \pm 0.17$ & $0.04^{*}$ \\
MFI of CD59-positive cells & $17.0 \pm 2.3$ & $20.3 \pm 1.6$ & $<0.001^{*}$ \\
\hline
\end{tabular}

Values are the mean \pm SD, $n(\%)$, or median (IQR). MFI, mean fluorescence intensity; RBCs, red blood cells. * Significant difference.

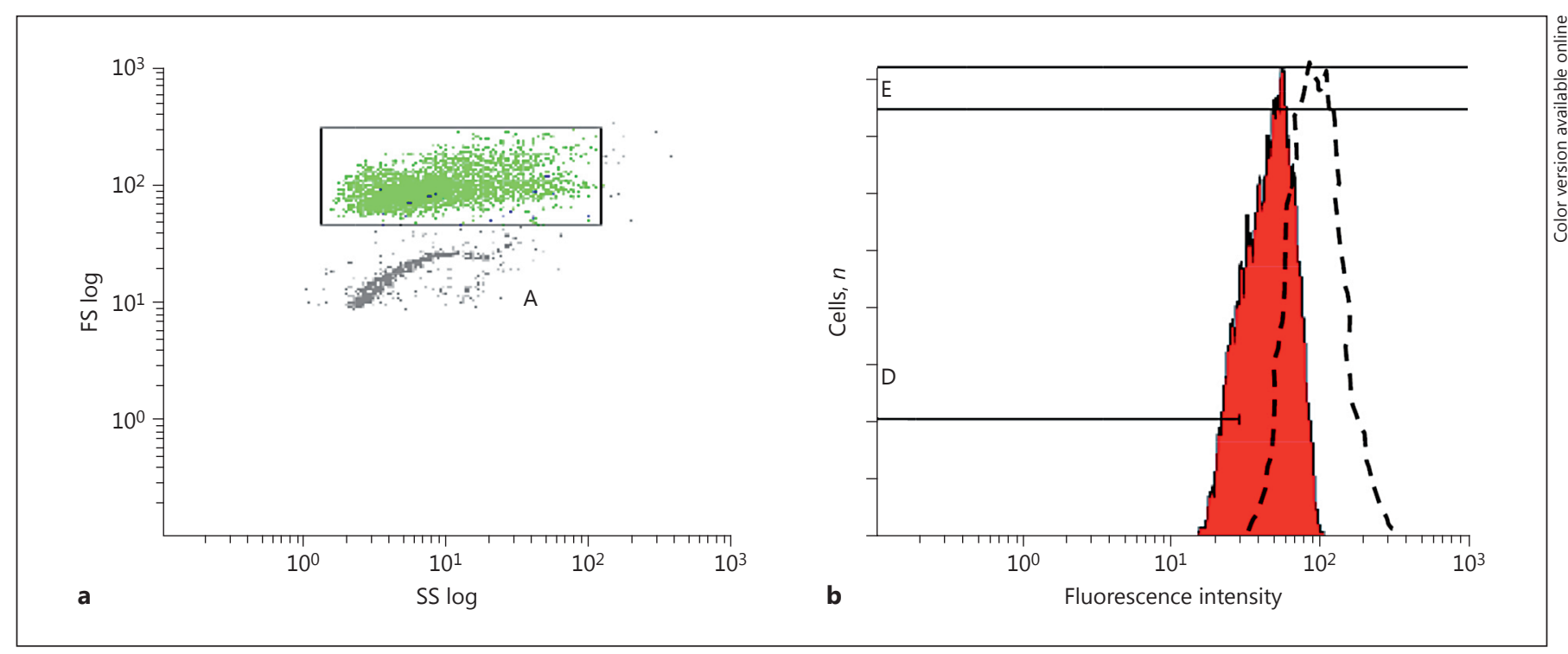

Fig. 1. a Representative dot plot showing RBC distribution and gating by forward scatter (FS) and side scatter (SS). b Representative histogram of an altered expression of CD59 on RBCs.

\section{Results}

The mean age of the patients was $56.6 \pm 13$ years, the mean hemoglobin level was $105.2 \pm 12.3 \mathrm{~g} / \mathrm{L}$, and the median serum creatinine level was $546 \mu \mathrm{mol} / \mathrm{L}$ (range 380869). The hematologic and biochemical data for the CKD patients and HCs are summarized in Table 1.

\section{Expression of CD55 and CD59 on RBCs of All Patients} with CKD

The number of cases $(n=68)$ with CD59 deficiency (deficient RBCs $>3 \%$ ) was significantly higher among CKD patients compared with HC subjects. CD55 deficiency was not detected in either patients or HCs (Ta- ble 2). The proportions of CD55- and CD59-deficient RBCs among CKD patients were significantly higher than among HCs $(p<0.001$; Table 2). The MFI of CD55 and CD59 expression on RBCs in patients with CKD was significantly less than the expression on RBCs in HCs ( $p<$ 0.04 and $p<0.001$, respectively; Table 2 ). A representative dot plot and histogram of the obtained resultant CD59 expression analysis in a CD59-deficent CKD patient is shown in Figure 1.

\section{Associations of CD55 and CD59 Expression among All CKD Patients}

The level of serum creatinine in patients with CKD showed a significant direct correlation with the propor- 
Table 3. Comparison between CD55 and CD59 expression patterns among CKD patient groups

\begin{tabular}{|c|c|c|c|c|}
\hline Parameters & $\begin{array}{l}\text { Group A } \\
(n=30)\end{array}$ & $\begin{array}{l}\text { Group B } \\
(n=30)\end{array}$ & $\begin{array}{l}\text { Group C } \\
(n=31)\end{array}$ & $p$ \\
\hline $\mathrm{Hb}, \mathrm{g} / \mathrm{L}$ & $104.0 \pm 14.9$ & $105.6 \pm 14.1$ & $105.9 \pm 6.5$ & 0.82 \\
\hline $\mathrm{LDH}, \mathrm{IU} / \mathrm{L}$ & $229 \pm 64$ & $215 \pm 99$ & $173 \pm 30$ & $0.007^{*}$ \\
\hline $\mathrm{CRP}, \mathrm{mg} / \mathrm{L}$ & $5.0(4.0-20.3)$ & $7.8(5.4-75.8)$ & $22(4.6-32.4)$ & 0.37 \\
\hline CD59 deficiency & $23(76.7)$ & $16(53.3)$ & $29(93.5)$ & $0.001^{*}$ \\
\hline Proportion of CD55-deficient RBCs, \% & $0.42(0.13-0.88)$ & $0.44(0.13-0.50)$ & $0.33(0.33-0.35)$ & 0.06 \\
\hline Proportion of CD59-deficient RBCs, \% & $3.3(2.8-4.2)$ & $3.5(1.9-5.3)$ & $4.4(4.3-4.5)$ & 0.07 \\
\hline MFI of CD55-positive cells & $2.9 \pm 0.14$ & $2.7 \pm 0.19$ & $1.8 \pm 0.10$ & $0.04^{*}$ \\
\hline MFI of CD59-positive cells & $22.8 \pm 2.1$ & $21.6 \pm 2.0$ & $17.9 \pm 2.5$ & $0.03^{*}$ \\
\hline
\end{tabular}

Values are the mean $\pm \mathrm{SD}, n(\%)$ or median (IQR). CKD, chronic kidney disease; Hb, hemoglobin; MCV, mean cell volume; LDH, lactate dehydrogenase; CRP, C-reactive protein; EPO, erythropoietin; MFI, mean fluorescence intensity. * Significant difference.

tions of CD55- and CD59-deficient RBCs ( $r=0.33, p<$ 0.001 , and $r=0.36 p<0.001$, respectively), but not with the MFI of CD55 or CD59 expression. The levels of CD55 and CD59 expression, as evident by the MFI on RBCs of CKD patients, showed a significantly inverse correlation with the level of the inflammatory biomarker, CRP ( $r=-0.31, p=0.03$, and $r=-0.33, p=0.003)$, respectively. There was also a significant inverse correlation between the hemoglobin level and the proportion of CD55-deficient RBCs $(r=-0.37, p<0.001)$, and the proportion of CD59-deficient RBCs $(r=-0.22, p=0.02)$; however, a significant relation was not observed between hemoglobin levels and the MFI of CD55 or CD59 expression.

The linear regression analysis showed an independent association between CD55 and CD59 expression and markers of hemolysis, including the reticulocyte count and serum LDH level. The proportion of CD55-deficient RBCs and the MFI of CD55 expression significantly accounted for about $14 \%(p<0.001)$ and $6 \%(p=0.02)$ of the variation in hemoglobin levels, respectively. Neither the proportion of CD59-deficient RBCs nor the MFI of CD59 expression was associated with the hemoglobin level. The proportion of CD55-deficient RBCs and the MFI of CD55 expression significantly accounted for about $13 \%(p<0.001)$ and $30 \%(p<0.001)$ of the variation in reticulocyte count, respectively, and only the MFI of CD55 expression significantly accounted for about $6 \%$ $(p=0.01)$ of the variation in serum LDH level. Neither the proportion of CD59-deficient cells nor the MFI of CD59 expression on RBCs of CKD patients was correlated with the reticulocyte count or with the serum LDH level.

\section{Expression Pattern of CD55 and CD59 among the 3} Subgroups of CKD Patients

The expression patterns of CD55 and CD59 among patients with CKD are given in Table 3. All CKD patients had RBC populations with CD59 deficiency. The number of CD59-deficient patients was significantly different between the 3 groups of patients (76.6\% of group A, 53.3\% of group B, and $93.5 \%$ of group C). CD55 deficiency was not detected in any subgroup. The proportions of CD55and CD59-deficient RBCs were not significantly different among the CKD patient subgroups; however, a significant difference was present in the MFI of CD55 and the MFI of CD59 among the 3 subgroups.

\section{Discussion}

In this study, the number of CKD patients with CD59 deficiency was significantly higher compared to the HC cohort, and there were increased proportions of CD55and CD59-deficient RBCs among CKD patients with various disease stages compared to normal HCs. The strength of CD59 expression on RBCs in the CKD patients, as shown by MFI, was also significantly lower than that in HC subjects. In this study, it could be speculated that altered CD55 and CD59 on RBCs of CKD patients might play a role in the increased susceptibility to hemolysis, 
leading to the development of anemia in this group of patients. A mild degree of hemolysis in CKD was previously demonstrated; however, its exact mechanism remains unclear [13]. Patients with CKD and those on chronic hemodialysis have enhanced susceptibility to complement activation because of a decreased renal catabolism of complement factor D [14]. They are also frequently exposed to increased complement activation as a result of inflammatory processes, including the contact of blood with dialysis membranes in patients on dialysis [15]. The terminal sequence of complement activation is initiated after the cleavage of $\mathrm{C} 5$ and leads to the assembly of the membrane attack complex (C5b-9) on target cell membranes. This creates a transmembrane pore that is functionally expressed by increased ion permeability of the RBC membrane and leads to osmotic swelling and the lysis of RBCs [16]. In a similar study, Himmelfarb et al. [17] reported that, although RBCs from CKD patients were susceptible to C5b-9 deposition with subsequent RBC ghost formation, the increased uremic RBC susceptibility to complement-mediated lysis was not associated with an altered expression of CD55 and CD59 on RBCs of CKD patients; however, unlike this study, they did not investigate the proportions of CD55- and CD59-deficient cells in their cohort of patients compared to HC subjects. It is possible that the difference in the sensitivity of the flow cytometric method used in their study is the cause of the different findings observed in this study.

In this study, the finding that there was a significant inverse correlation between the proportions of CD55and CD59-deficient RBCs in CKD patients and hemoglobin levels, and a direct correlation with serum creatinine levels could be due to the susceptibility of RBCs in CKD patients to some degree of complement-mediated hemolysis that caused a multifactorial etiology of anemia in this group of patients. The significant inverse correlation between the inflammatory biomarker (CRP, and the MFI of CD55) and CD59 expression in this study could be due to the fact that patients with CKD were frequently exposed to active inflammatory processes that had been shown to be associated with loss of progressive renal function, and were etiologically related to several factors that included uremia, the release of cytokines by exposure to dialysis membrane, impaired renal clearance of cytokines, recurrent infections, and a reduced antioxidant capacity of plasma and RBCs $[18,19]$.

The preferential significant association between the proportion of CD55-deficient cells and MFI of CD55 expression in patients with CKD and markers of hemolysis, including the hemoglobin level, reticulocyte count, and

CD55 and CD59 Expression in Anemia of CKD serum LDH level, and not with CD59-deficinet RBCs, could be due to the possibility that CD55 is a more critical protective complement regulatory protein than CD59.

The significant association observed in this study between the strength of CD55 and CD59 expression, as evident by their MFI, and EPO therapy is consistent with the findings of Ohi et al. [12], who showed that recombinant EPO therapy increased the expression of CD55 and CD59 on RBCs of anemic patients on hemodialysis, thus mediating its efficacy. This study also showed that the serum CRP level, which was previously shown to be raised in CKD patients, negatively influenced the level of CD55 and CD59 expression [18]. It was previously postulated to explain the relative EPO resistance in about $25 \%$ of CKD patients on RRT [18].

In agreement with previous studies, the results of this study suggest that altered expression of CD55 and CD59 on RBCs is not specific to PNH [20,21]. It has been described in several disorders, including autoimmune hemolytic anemia, HIV infection, type 2 diabetes, and macrovascular disease, systemic lupus erythematosus, autoimmune thrombocytopenia, aplastic anemia, and polytransfused patients with $\beta$-thalassemia major [22-26].

The preferential deficiency of CD59 in CKD patients, compared to CD55, is of interest. A similar finding was observed in a sickle cell disease patient [27]. The molecular basis of this finding is unclear, as it is unlikely to be due to a defective biosynthesis of the common GPI-anchored membrane proteins that is seen in PNH stem cell clones [28]. One possible explanation is related to the specificity of the gating strategy that is used in this study, which relies upon simple light scatter to "gate" RBCs in the sample. It had been shown that the specificity of RBC isolation could be improved by the addition of antibodies to glycophorin A (CD235a), a mucin-like transmembrane protein. It allows a more specific distinction of RBCs from cells of other lineages and debris, which may otherwise be misinterpreted as deficient RBCs [29].

\section{Conclusion}

This study has shown that the pattern of CD55 and CD59 expression on RBCs was altered in anemic CKD patients. This finding could be a contributory factor in the pathophysiology of anemia in CKD, and could be influenced by the patient's inflammatory status and serum EPO level. Further studies are needed to explore the mechanism that alters CD55 and CD59 expression on RBCs in this group of patients. 


\section{Acknowledgments}

We would like to thank Mrs. Suhair Al-Fawal and Mrs. Sicy Easow for their technical assistance. We would also like to thank the Research Core Facility of the Health Sciences Center for the use of the flow cytometric analyzer, which is supported by projects GM $01 / 01$ and GM 01/05. The work was partly supported by a grant from the Research Administration, Kuwait University (MG 04/13).

\section{Disclosure Statement}

The authors declare that they have no conflicts of interest to disclose.

\section{References}

1 McClellan WM, Jurkovitz C, Abramson J: The epidemiology and control of anaemia among pre-ESRD patients with chronic kidney disease: Eur J Clin Invest 2005;3:58-65.

2 McClellan W, Aronoff SL, Bolton WK, et al: The prevalence of anemia in patients with chronic kidney disease. Curr Med Res Opin 2004;9:1501-1510.

3 McGonigle RJ, Wallin JD, Shadduck RK, et al: Erythropoietin deficiency and inhibition of erythropoiesis in renal insufficiency. Kidney Int 1984;25:437-444.

4 Babitt J, Lin H: Mechanisms of anemia in CKD. J Am Soc Nephrol 2012;23:1631-1634.

5 Meytes D, Bogin E, Ma A, et al: Effect of parathyroid hormone on erythropoiesis. J Clin Invest 1981;67:1263-1269.

6 Ly J, Marticorena R, Donnelly S: Red blood cell survival in chronic renal failure. Am J Kidney Dis 2004;44:715-719.

7 Christopher MM: Erythrocyte morphologic changes linked to biochemical abnormalities. Proceedings of the XI Congress, International Society for Animal Biochemistry, Valdivia, Chile, 2004, pp 117-121.

8 Wu SG, Jeng FR, Wei SY, et al: Red blood cell osmotic fragility in chronically hemodialyzed patients. Nephron 1998;78:28-32.

9 Holguin $\mathrm{MH}$, Fredrick LR, Bernshaw NJ, et al: Isolation and characterization of a membrane protein from normal human erythrocytes that inhibits reactive lysis of the erythrocytes of paroxysmal nocturnal hemoglobinuria. J Clin Invest 1989;84:7-17.

10 Meri S, Jarva H: Complement regulation. Vox Sang 1998;74(suppl 2):291-302.

11 Lublin D, Atkinson J: Decay accelerating factor: biochemistry, molecular biology and function. Ann Rev Immunol 1989;7:35-58.

12 Ohi H, Tamano M, Sudo S, et al: Recombinant EPO therapy increases erythrocyte expression of complement regulatory proteins. Am J Kidney Dis 2003;41:179-185.
13 Eschbach J: The anemia of chronic renal failure: pathophysiology and the effects of recombinant erythropoietin. Kidney Int 1989; 35:134-148.

14 Pascual M, Steiger G, Estreicher J, et al: Metabolism of complement factor $D$ in renal failure. Kidney Int 1988;34:529-536.

15 Volanakis JE, Barnum SR, Giddens M, et al: Renal filtration and catabolism of complement protein D. N Engl J Med 1985;312:395399.

16 Podack ER: Assembly and structure of the membrane attack (MAC) of complement; in Podack ER (ed): Cytologic Lymphocytes and Complement Effectors of the Immune System. Boca Raton, CRC Press, 1988, p 173.

17 Himmelfarb J, Mcmonagle E, Holbrook D, et al: Increased susceptibility to erythrocyte C5b-9 deposition and complement-mediated lysis in chronic renal failure. Kidney Int 1999; 55:659-666.

18 Shlipak MG, Fried LF, Crump C, et al: Elevations of inflammatory and procoagulant biomarkers in elderly persons with renal insufficiency. Circulation 2003;107:87-92.

19 Bolton CH, Downs LG, Victory JG, et al: Endothelial dysfunction in chronic renal failure: roles of lipoprotein oxidation and pro-inflammatory cytokines. Nephrol Dial Transplant 2001;16:1189-1197.

20 Nebe C: Paroxysmal nocturnal hemoglobinuria: clinical aspects and flow cytometric analysis of red blood cells. Clin Lab 1997; 81-94.

21 Ruiz-Delgado GJ, Vázquez-Garza E, MéndezRamírez N, et al: Abnormalities in the expression of CD55 and CD59 surface molecules on peripheral blood cells are not specific to paroxysmal nocturnal hemoglobinuria. Hematology 2009;14:33-37.
22 Barros M, Yamamoto M, Figueiredo M, et al: Expression levels of CD47, CD35, CD55, and CD59 on red blood cells and signal-regulatory protein on monocytes from patients with warm autoimmune hemolytic anemia. Transfusion 2009;49:154-160.

23 Terpos E, Sarantopoulos A, Kouramba A, et al: Reduction of CD55 and/or CD59 in red blood cells of patients with HIV infection. Med Sci Monit 2008;14:276-280.

24 Xi-wen M, Zhi-wen C, Ming-zhao Q, et al: Decreased expression of complement regulatory proteins, CD55 and CD59, on peripheral blood leucocytes in patients with type 2 diabetes and macrovascular diseases. Chin Med J 2009;122:2123-128.

25 Alegretti AP, Mucenic T, Merzoni J, et al: Expression of CD55 and CD59 on peripheral blood cells from systemic lupus erythematosus (SLE) patients. Cell Immunol 2010;265: 127-132.

26 Salama MA, Sadek NA, Hassab HM, et al: Erythrocyte autoantibodies and expression of CD59 on the surface of red blood cells of polytransfused patients with $\beta$-thalassaemia major. Br J Biomed Sci 2004;61:88-92.

27 Al-Faris L, Al-Rukhayes M, Al-Humood S: Expression pattern of CD55 and CD59 on red blood cells in sickle cell disease. Hematology 2017;22:105-113.

28 Meletis J, Terpos E: Recent insights into the pathophysiology of paroxysmal nocturnal hemoglobinuria. Med Sci Monit 2003;9:161172.

29 Borowitz MJ, Craig FE, DiGiuseppe JA, Illingworth AJ, et al: Guidelines for the diagnosis and monitoring of paroxysmal nocturnal hemoglobinuria and related disorders by flow cytometry. Cytometry B Clin Cytom 2010;78: 211-230. 